\title{
ESTIMATION FOR TWO-WAY ANALYSIS OF VARIANCE WITH CORRELATED ERRORS
}

\author{
Alasford M. Ngwengwe \\ and \\ Charles E. McCulloch \\ Biometrics Unit and Statistics Center \\ Cornell University, Ithaca, New York 14853, U.S.A.
}




\title{
ESTIMATION FOR TWO-WAY ANALYSIS OF VARIANCE \\ WITH CORRELATED ERRORS
}

\author{
Alasford M. Ngwengwe \\ and \\ Charles E. McCulloch
}

Biometrics Unit and Statistics Center

Cornell University, Ithaca, New York 14853, U.S.A.

Key Words and Phrases: correlated errors; intraclass correlation coefficient; log-likelihood function; maximum likelihood estimator; mean squares; mixed effects model; multivariate normal; uniform covariance structure.

\begin{abstract}
We consider some estimation and distribution problems encountered in a two way analysis of variance model with only one observation per cell, errors correlated in one level, and the variances are not necessarily equal. The independence criteria for the row and interaction mean sum of squares and distribution of the maximum likelihood estimator of the correlation coefficient are given.
\end{abstract}

\section{INTRODUCTION}

The topics considered in this paper were motivated by a problem in ecology with density data on species. Consider M species (or, more generally, columns) under study in $\mathrm{N}$ sites (or rows) and let $\mathrm{Y}_{\mathrm{ij}}$ be the density of the $\mathrm{j}^{\text {th }}$ species in the $\mathrm{i}^{\text {th }}$ site. Because of coexistence of the species in a site, it can be assumed that observations in the same site are correlated. We assume that $Y_{i j}$ can be mathematically described as

$$
\mathrm{Y}_{\mathrm{ij}}=\mu+\beta_{\mathrm{i}}+\alpha_{\mathrm{j}}+\epsilon_{\mathrm{ij}}
$$

for $\mathrm{i}=1,2, \cdots, \mathrm{N}$, and $\mathrm{j}=1,2, \cdots, \mathrm{M}$, where $\mathrm{Y}_{\mathrm{ij}}$ is the measurement for the $\mathrm{j}^{\text {th }}$ column in the $\mathrm{i}^{\text {th }}$ row; $\mu$ is the overall mean, constant for all columns on all 
rows; $\beta_{\mathrm{i}}$ is the effect of the $\mathrm{i}^{\text {th }}$ row; $\alpha_{\mathrm{j}}$ is the effect of the $\mathrm{j}^{\text {th }}$ column, and $\epsilon_{\mathrm{ij}}$ is the random error associated with the $j^{\text {th }}$ column on the $i^{\text {th }}$ row. A key aspect of the ecology problem was to measure the overall association among the species (columns).

The model (1.1) differs from the usual analysis of variance model in that we take errors in a column to be homoscedastic, but the variance may change from column to column and the columns are correlated. Let $\underline{E}_{i}$ be the $M \times 1$ vector of errors in the $\mathrm{i}^{\text {th }}$ row. We assume that the $\mathrm{E}_{\mathrm{i}}$ 's are independently and identically distributed as multivariate normal with zero mean and positivedefinite covariance matrix, $\Sigma$.

This model has been considered by Olkin and Vaeth (1981), Andersen, Jensen and Schou (1981), and Box (1954). Box (1954) and Andersen, Jensen and Schou (1981) consider distributions of the usual two-way analysis of variance test statistics and Olkin and Vaeth (1981) give the maximum likelihood estimators for row and column parameters and the covariance matrix for the case where there are $K(>1)$ replications in each cell. We consider the case with a single replication per cell and focus on measures of association between the columns. In Section 2 we derive conditions for independence of the mean squares, correcting an error in Andersen, Jensen and Schou (1981). In Section 3 we consider maximum likelihood estimation under various covariance structures and in Section 4 we derive the distribution, mean and variance of the maximum likelihood estimator of the common correlation.

\section{INDEPENDENCE OF THE MEAN SQUARES}

We determine when the mean squares are independent. We are particularly interested in three covariance structures which are relevant to our application; namely, uniform covariance, unequal correlations with equal variances, and unequal variances with equal correlation. Let MSRows, MSCols, and MSInt denote the row, column, and interaction mean sum of squares respectively from a two-way analysis of variance (rows by columns). Let $\mathrm{J}_{\mathrm{n}}$ denote an $n \times n$ matrix of ones and $\bar{J}_{n}=J_{n} / n$ and let $C_{n}=I_{n}-\bar{J}_{n}$ denote the centering matrix of order $n$. Then the mean squares can be written as follows:

$$
\begin{aligned}
& \text { MSRows }=\left[\mathrm{Y}^{\mathrm{t}} \mathrm{AY}\right] /(\mathrm{N}-1) \quad \text { where } \mathrm{A}=\mathrm{C}_{\mathrm{N}} \otimes \overline{\mathrm{J}}_{\mathrm{M}} \text {, } \\
& \text { MSCols }=\left[\mathrm{Y}^{\mathrm{t}} \mathrm{BY}\right] /(\mathrm{M}-1) \quad \text { where } \mathrm{B}=\overline{\mathrm{J}}_{\mathrm{N}} \otimes \mathrm{C}_{\mathrm{M}} \text {, }
\end{aligned}
$$




$$
\text { MSInt }=\left[\mathrm{Y}^{\mathrm{t}} \mathrm{HY}\right] /(\mathrm{N}-1)(\mathrm{M}-1) \text { where } \mathrm{H}=\mathrm{C}_{\mathrm{N}} \otimes \mathrm{C}_{\mathrm{M}}
$$

and $\mathrm{A} \otimes \mathrm{B}$ is the Kronecker product of $\mathrm{A}$ and $\mathrm{B}$ (Searle, 1984).

Two quadratic forms $Y^{t} P Y$ and $Y^{t} Q Y$ are independent if and only if $P V Q$ is zero, where $\mathrm{V}$ is the variance-covariance matrix of $\mathrm{Y}$, when $\mathrm{Y}$ has a multivariate normal distribution (Craig's theorem, Craig 1943; see also Searle, 1971, pp 59-64). We have AVB $=\mathrm{C}_{\mathrm{N}} \overline{\mathrm{J}}_{\mathrm{N}} \otimes \overline{\mathrm{J}}_{\mathrm{M}} \Sigma \overline{\mathrm{J}}_{\mathrm{M}}$ and $\mathrm{BVH}=$ $\overline{\mathrm{J}}_{\mathrm{N}} \mathrm{C}_{\mathrm{N}} \otimes \mathrm{C}_{\mathrm{M}} \Sigma \mathrm{C}_{\mathrm{M}}$, which are both zero since $\mathrm{C}_{\mathrm{k}} \mathrm{J}_{\mathrm{k}}=\mathrm{J}_{\mathrm{k}} \mathrm{C}_{\mathrm{k}}=0$, that is, MSRows and MSCols are independent and so are MSCols and MSInt for any positivedefinite covariance matrix $\Sigma$. For the row and interaction mean squares we have:

$$
\begin{aligned}
\mathrm{AVH} & =\left[\mathrm{C}_{\mathrm{N}} \otimes \overline{\mathrm{J}}_{\mathrm{M}}\right]\left[\mathrm{I}_{\mathrm{N}} \otimes \Sigma\right]\left[\mathrm{C}_{\mathrm{N}} \otimes \mathrm{C}_{\mathrm{M}}\right] \\
& =\mathrm{C}_{\mathrm{N}} \otimes \overline{\mathrm{J}}_{\mathrm{M}}[\Sigma-\mathrm{dI} \mathrm{M}],
\end{aligned}
$$

where

$$
\mathrm{d}=\frac{1}{\mathrm{M}} \sum_{\mathrm{j}=1}^{\mathrm{M}} \sum_{\mathrm{u}=1}^{\mathrm{M}} \rho_{\mathrm{ju}} \sigma_{\mathrm{j}} \sigma_{\mathrm{u}}
$$

where $\rho_{\mathrm{jk}}$ is the correlation between the $\mathrm{j}^{\text {th }}$ and $\mathrm{k}^{\text {th }}$ columns, and $\sigma_{\mathrm{j}}$ is the standard deviation for the $\mathrm{j}^{\text {th }}$ column. From equation (2.1), MSRows and MSInt are independently distributed if and only if

$$
\text { (a) } \mathrm{J}_{\mathrm{M}} \Sigma=\mathrm{J}_{\mathrm{M}^{\mathrm{d}}} \quad \text { or (b) } \sum_{\mathrm{j}=1}^{\mathrm{M}} \rho_{\mathrm{jk}} \sigma_{\mathrm{j}} \sigma_{\mathrm{k}}=\mathrm{d}
$$

for all $\mathrm{k}$, where $\mathrm{k}=1,2, \cdots, \mathrm{M}$. That is, MSRows and MSInt are independently distributed if and only if the column (row) totals of the $\Sigma$ are all equal. Taking transposes on both sides of (2.3) (a), we have $\Sigma \mathrm{J}_{M}=\mathrm{dJ}_{M}$ which is satisfied if and only if $\Sigma 1_{M}=\mathrm{d}_{1}$. Expressed differently, MSRows and MSInt are independently distributed if and only if d defined in (2.2) is an eigenvalue of $\Sigma$ with the vector of ones as the corresponding eigenvector. This is a correction to Andersen, Jensen and Schou (1981)'s theorem 3.1 part (ii), which states that MSRows and MSInt are independently distributed when the variances are equal for $\mathrm{M} \geq 2$, if and only if we have equal correlation structure. Below we give examples for $M=4$ where variances are equal, but not all the correlations are equal and yet the independence criteria for MSRows and MSInt is satisfied.

If the variances are all equal to $\sigma^{2}$ but the correlations are not necessarily all equal, then $\Sigma$ is $\sigma^{2} \mathrm{P}$, where $\mathrm{P}$ is the correlation matrix. Let $\bar{\rho} .$. denote the 
average of the $\mathrm{M}(\mathrm{M}-1) / 2$ unique correlations and let $\bar{\rho}_{\cdot \mathrm{j}}$ denote the average of the M-1 correlations in the $\mathrm{j}^{\text {th }}$ column (row) of $\mathrm{P}$. We have $\mathrm{d}=$ $[1+(M-1) \bar{\rho} ..] \sigma^{2}$ and condition (2.3) for the independence of MSRows and MSInt reduces to $\bar{\rho}_{\cdot \mathrm{j}}=\bar{\rho} .$. , for all $\mathrm{j}=1,2, \cdots, \mathrm{M}$; therefore MSRows and MSInt are independent if and only if all the column (row) average correlations are equal. For $\mathrm{M}=3$, the independence criterion is satisfied only for the equal correlation situation and for $\mathrm{M}=4$, the equation is satisfied only for correlation matrices $\mathrm{P}^{*}$ of the form given below

$$
\mathrm{P}^{*}=\left[\begin{array}{cccc}
1 & \rho_{1} & \rho_{2} & \rho_{3} \\
\rho_{1} & 1 & \rho_{3} & \rho_{2} \\
\rho_{2} & \rho_{3} & 1 & \rho_{1} \\
\rho_{3} & \rho_{2} & \rho_{1} & 1
\end{array}\right]
$$

where $\rho_{1}, \rho_{2}, \rho_{3} \epsilon(-1,1)$ such that $\mathrm{P}^{*}$ is positive-definite. A matrix of the form of $\mathrm{P}^{*}$ is known as a centro-symmetric matrix (see Graybill, 1983, p. 287). An example of a special case of $\mathrm{P}^{*}$ is $\rho_{1}=\rho_{3}=\rho$, and $\rho_{2}=\rho^{2}$, which is positive definite for any $\rho \epsilon(-1,1)$. If the correlations are equal but the variances are not all equal, it can be shown that the independence condition is satisfied only when the variances are all equal if $\rho \geq 0$. With a covariance structure such that (2.3) is satisfied, MSRows/MSInt is a ratio of independent variables whose exact or approximate distribution is easier to work with than when MSRows and MSInt are not independent. We will use the results in Section 4.2.

\section{MAXIMUM LIKELIHOOD ESTIMATION}

In this section we discuss maximum likelihood estimation under several covariance structures. In Section 3.1 we consider models with block effects. Section 3.2 considers a model with no block effects and with an equal correlation between columns.

\subsection{Block effects models with uniform covariance matrix}

We first consider the simple model with only block effects, i.e., $\mathrm{Y}_{\mathrm{ij}}=\beta_{\mathrm{i}}+$ $\epsilon_{\mathrm{ij}}$ with $\Sigma=\operatorname{Var}\left(\epsilon_{\mathrm{i}}\right)$. When $\Sigma$ is the uniform covariance matrix, i.e., equal 
variance and equal correlation, it is straightforward to show that the likelihood is unbounded and the maximum likelihood estimators do not exist. This is intuitively clear since one way to generate the uniform covariance model is through

$$
\begin{aligned}
\epsilon_{\mathrm{ij}} & =\delta_{\mathrm{i}}+\eta_{\mathrm{ij}}, \\
\delta_{\mathrm{i}} & \sim \operatorname{iid~} \mathrm{N}\left(0, \sigma_{\delta}^{2}\right) \\
\eta_{\mathrm{ij}} & \sim \operatorname{iid~} \mathrm{N}\left(0, \sigma_{\eta}^{2}\right) .
\end{aligned}
$$

It is then clear that the $\beta_{\mathrm{i}}$ and $\delta_{\mathrm{i}}$ effects cannot be separated, and hence that the $\beta_{\mathrm{i}}$ and $\Sigma$ with a uniform covariance structure cannot be separately estimated.

Therefore, the claim by Olkin and Vaeth (1981) that we can estimate all the variances and covariances for this particular patterned covariance matrix is not true. We cannot estimate any of the covariance parameters if the block effects are not known. However, the problem does not arise when there are replicates in each cell as pointed out by Olkin and Vaeth (1981).

In the site-by-species data, the sites (rows) may be a random sample of sites. A suitable model for analyzing the data is then the two-way classification model with $\beta_{\mathrm{i}}$ 's being random. We therefore have

$$
\begin{gathered}
\mathrm{Y}_{\mathrm{ij}}=\mu+\beta_{\mathrm{i}}+\alpha_{\mathrm{j}}+\epsilon_{\mathrm{ij}} \\
\beta_{\mathrm{i}} \sim \text { iid } \mathrm{N}\left(0, \sigma_{\beta}^{2}\right) \\
\underline{\mathrm{E}}_{\mathrm{i}} \sim \text { iid } \mathrm{N}_{\mathrm{M}}(0, \Sigma) . \\
\beta_{\mathrm{i}} \text { independent of } \mathrm{E}_{\mathrm{i}} .
\end{gathered}
$$

By the same reasoning as above, the parameters for this model also cannot be separately estimated when $\Sigma$ follows the uniform covariance structure.

\subsection{No block effects with equal correlation but unequal variances}

Suppose we have equal correlation, but the variances are not all equal. We consider the one-way analysis of variance model (1.1) without block effects, i.e., $\underline{\beta}=0$. Then $2 \mathrm{~N}^{-1}$ times the log-likelihood function of $\sigma_{1}^{2}, \sigma_{2}^{2}, \cdots, \sigma_{\mathrm{M}}^{2}$, and $\rho$, with $\mu+\alpha_{j}$ 's substituted by their maximum likelihood estimators, $\bar{Y}_{. j}$ 's, and ignoring constants is given by 


$$
\begin{aligned}
& \mathrm{L}=-(\mathrm{M}-1) \log [1-\rho]-\log [1+(\mathrm{M}-1) \rho] \\
&-\sum_{\mathrm{j}=1}^{\mathrm{M}} \log \left[\sigma_{\mathrm{j}}^{2}\right]-\frac{1+(\mathrm{M}-2) \rho}{[1-\rho][1+(\mathrm{M}-1) \rho]} \sum_{\mathrm{j}=1}^{\mathrm{M}} \frac{\mathrm{S}_{\mathrm{j}}^{2}}{\sigma_{\mathrm{j}}^{2}} \\
&+\frac{\rho}{[1-\rho][1+(\mathrm{M}-1) \rho]} \sum_{\mathrm{j} \neq \mathrm{k}}^{\mathrm{M}} \sum^{\mathrm{M}} \frac{\mathrm{r}_{\mathrm{jk}} \mathrm{S}_{\mathrm{j}} \mathrm{S}_{\mathrm{k}}}{\sigma_{\mathrm{j}} \sigma_{\mathrm{k}}}
\end{aligned}
$$

where $\mathrm{S}_{\mathrm{j}}^{2}$ is the sample variance of the $\mathrm{j}^{\text {th }}$ column using divisor $\mathrm{N}$ instead of $\mathrm{N}-1$ and $r_{j k}$ is the sample correlation between the $j^{\text {th }}$ and $k^{\text {th }}$ columns. After equating to zero the derivative of $\mathrm{L}$ with respect to $\rho$ and multiplying through by $(1-\hat{\rho})^{2}[1+(M-1) \hat{\rho}]^{2}$ we have the following:

$$
\hat{\rho}[2+(\mathrm{M}-2) \hat{\rho}][1-\mathrm{Q}(\hat{\theta})]=\left[1+(\mathrm{M}-1) \hat{\rho}^{2}\right][\hat{\rho}-\overline{\mathbf{r}}(\hat{\theta})]
$$

where $\hat{\theta}_{\mathrm{j}}^{2}=\mathrm{S}_{\mathrm{j}}^{2} / \hat{\sigma}_{\mathrm{j}}^{2}$,

$$
\mathrm{Q}(\hat{\theta})=\frac{1}{\mathrm{M}} \sum_{\mathrm{j}=1}^{\mathrm{M}} \hat{\theta}_{\mathrm{j}}^{2}, \quad \text { and } \quad \overline{\mathbf{r}}(\hat{\theta})=\frac{1}{\mathrm{M}(\mathrm{M}-1)} \sum_{\mathrm{j} \neq \mathrm{k}}^{\mathrm{M}} \sum_{\mathrm{j}}^{\mathrm{M}} \mathrm{r}_{\mathrm{j}} \hat{\theta}_{\mathrm{j}} \hat{\theta}_{\mathrm{k}},
$$

Next differentiating $L$ with respect to $\sigma_{j}^{2}(j=1,2, \cdots, M)$, equating the resulting expression to zero, multiplying each equation by $\sigma_{\mathrm{j}}^{2}$ and adding them and multiplying through by $\hat{\rho}(1-\hat{\rho})[1+(\mathrm{M}-1) \hat{\rho}] / \mathrm{M}$ we get:

$$
\mathrm{M}[1+(\mathrm{M}-2) \hat{\rho}] \mathrm{Q}(\hat{\theta})=\mathrm{M}[1-\hat{\rho}][1+(\mathrm{M}-1) \hat{\rho}]+\hat{\rho} \overline{\mathbf{r}}(\hat{\theta})
$$

We show that $\mathrm{Q}(\hat{\theta})=1$ by taking the difference of (3.2.3) multiplied by $\hat{\rho}$ and (3.2.3) multiplied by $\left[1+(M-1) \hat{\rho}^{2}\right]$. Upon simplification we get

$$
[1-\hat{\rho}][1+(\mathrm{M}-1) \hat{\rho}] \mathrm{Q}(\hat{\theta})=[1-\hat{\rho}][1+(\mathrm{M}-1) \hat{\rho}]
$$

hence the result. From equation (3.2.2) and the above result we get

$$
\hat{\rho}_{\text {mle }}=\frac{1}{M(M-1)} \sum_{j \neq k}^{M} \sum_{j k}^{M} r_{j} \hat{\theta}_{j} \hat{\theta}_{k} .
$$

That is, there are no closed form solutions for the maximum likelihood estimator of the common correlation and the variances.

A simulation study was done to compare $\hat{\rho}_{\text {mle }}$, the maximum likelihood estimator for $\rho$, and the average sample correlation $\overline{\mathrm{r}} \ldots \mathrm{M}$ was set equal to 3 , with two variance structures $\mathrm{V}_{1}=(2,4,6)$ and $\mathrm{V}_{2}=(2,2,8)$. Table I gives average values of $\hat{\rho}_{\text {mle }}$ and $\bar{r}$.. along with the standard errors (s.e) and square root of the mean squares errors (RMSE) for the eleven equal correlation 
structures. The results of the simulation show that the two estimators are equivalent for most values of $\rho$. That is, for most cases there is no need to compute the maximum likelihood estimator of $\rho$, the average of the sample correlations is adequate.

To investigate the large sample behavior of $\hat{\rho}_{\text {mle }}$ and $\overline{\mathbf{r}} .$. we checked to see if they were asymptotically equivalent. That is we checked if the limiting distribution of $\mathrm{N}^{1 / 2}\left(\hat{\rho}_{\text {mle }}-\overline{\mathrm{r}}_{. .}\right)$converged to zero in probability. It can be shown that the two estimators are asymptotically equivalent if and only if

$$
\mathrm{N}^{1 / 2}\left[\frac{\mathrm{S}_{\mathrm{j}} \mathrm{S}_{\mathrm{k}}}{\hat{\hat{\sigma}}_{\mathrm{j}} \hat{\sigma}_{\mathrm{k}}}-1\right] \stackrel{\mathrm{pr}}{\rightarrow} 0, \quad \text { as } \mathrm{N} \rightarrow \infty .
$$

Condition (3.2.6) is satisfied if the asymptotic variances of $S_{j}$ and that of $\hat{\sigma}_{j}$ are equal. The difference between the asymptotic variances of $S_{j}$ and $\hat{\sigma}_{j}$ after dividing by $\sigma_{\mathrm{j}}^{2}$ is

$$
\frac{(\mathrm{M}-1)(\mathrm{M}-2) \rho^{2}[1-\rho]^{2}}{2 \mathrm{M}\left[2[1-\rho][1+(\mathrm{M}-1) \rho]+\mathrm{M} \rho^{2}\right]} \text {. }
$$

It follows that $\mathrm{N}^{1 / 2}\left(\hat{\rho}_{\text {mle }}-\overline{\mathrm{r}}_{\text {.. }}\right)$ fails to converge to zero in probability unless $\rho$ $=0$ or 1 . However, the difference (3.2.7) is practically zero for most values of $\rho$ corroborating the simulation results; see Figure I. That is, for all practical purposes the average of the sample correlations is adequate as an estimate of the common correlation. 
TABLE I

The average maximum likelihood estimator, $\hat{\rho}_{\text {mle }}$ and the average sample correlation, $\overline{\mathbf{r}}$, with their standard errors (s.e) and root mean square errors (RMSE) for eleven equal correlation structures. 1,000 trials with sample size 21 for each trial, $\mathrm{V}_{1}$ and $\mathrm{V}_{2}$ are variance structures.

$$
\mathrm{v}_{1}(2,4,6) \quad \mathrm{v}_{2}(2,2,8)
$$

\begin{tabular}{|c|c|c|c|c|}
\hline$\rho$ & $\begin{array}{c}\text { ave. } \overline{\mathbf{r}} \\
\text { s.e } \\
\text { RMSE } \\
\end{array}$ & $\begin{array}{c}\text { ave. } \hat{\rho}_{\text {mle }} \\
\text { s.e } \\
\text { RMSE }\end{array}$ & $\begin{array}{l}\text { ave. } \overline{\mathbf{r}} \\
\text { s.e } \\
\text { RMSE }\end{array}$ & $\begin{array}{c}\text { ave. } \hat{\rho}_{\text {mle }} \\
\text { s.e } \\
\text { RMSE } \\
\end{array}$ \\
\hline-0.48 & $\begin{array}{r}-0.4712 \\
0.0004 \\
0.0153\end{array}$ & $\begin{array}{r}-0.4801 \\
0.0003 \\
0.0082\end{array}$ & $\begin{array}{r}-0.4714 \\
0.0004 \\
0.0162\end{array}$ & $\begin{array}{r}-0.4801 \\
0.0003 \\
0.0094\end{array}$ \\
\hline-0.40 & $\begin{array}{r}-0.3913 \\
0.0012 \\
0.0391\end{array}$ & $\begin{array}{r}-0.4011 \\
0.0012 \\
0.0373\end{array}$ & $\begin{array}{r}-0.3934 \\
0.0012 \\
0.0392\end{array}$ & $\begin{array}{r}-0.4032 \\
0.0012 \\
0.0383\end{array}$ \\
\hline-0.20 & $\begin{array}{r}-0.1943 \\
0.0029 \\
0.0932\end{array}$ & $\begin{array}{r}-0.0982 \\
0.0030 \\
0.0954\end{array}$ & $\begin{array}{r}-0.2002 \\
0.0029 \\
0.0934\end{array}$ & $\begin{array}{r}-0.2051 \\
0.0030 \\
0.0962\end{array}$ \\
\hline-0.10 & $\begin{array}{r}-0.0963 \\
0.0036 \\
0.1132\end{array}$ & $\begin{array}{r}-0.0983 \\
0.0036 \\
0.1153\end{array}$ & $\begin{array}{r}-0.1032 \\
0.0036 \\
0.1131\end{array}$ & $\begin{array}{r}-0.1061 \\
0.0037 \\
0.1162\end{array}$ \\
\hline 0.00 & $\begin{array}{l}0.0031 \\
0.0040 \\
0.1281\end{array}$ & $\begin{array}{l}0.0022 \\
0.0041 \\
0.1313\end{array}$ & $\begin{array}{r}-0.0061 \\
0.0040 \\
0.1294\end{array}$ & $\begin{array}{r}-0.0073 \\
0.0041 \\
0.1312\end{array}$ \\
\hline 0.20 & $\begin{array}{l}0.1994 \\
0.0046 \\
0.1463\end{array}$ & $\begin{array}{l}0.2001 \\
0.0046 \\
0.1472\end{array}$ & $\begin{array}{l}0.1884 \\
0.0046 \\
0.454\end{array}$ & $\begin{array}{l}0.1903 \\
0.0046 \\
0.1461\end{array}$ \\
\hline 0.30 & $\begin{array}{l}0.2972 \\
0.0046 \\
0.1473\end{array}$ & $\begin{array}{l}0.2981 \\
0.0047 \\
0.1481\end{array}$ & $\begin{array}{l}0.2863 \\
0.0046 \\
0.1473\end{array}$ & $\begin{array}{l}0.2884 \\
0.0046 \\
0.1474\end{array}$ \\
\hline 0.70 & $\begin{array}{l}0.6931 \\
0.0032 \\
0.1031\end{array}$ & $\begin{array}{l}0.6932 \\
0.0032 \\
0.1023\end{array}$ & $\begin{array}{l}0.6874 \\
0.0031 \\
0.1001\end{array}$ & $\begin{array}{l}0.6883 \\
0.0031 \\
0.0994\end{array}$ \\
\hline 0.80 & $\begin{array}{l}0.7943 \\
0.0024 \\
0.0761\end{array}$ & $\begin{array}{l}0.7942 \\
0.0024 \\
0.0761\end{array}$ & $\begin{array}{l}0.7904 \\
0.0023 \\
0.0742\end{array}$ & $\begin{array}{l}0.7904 \\
0.0023 \\
0.0741\end{array}$ \\
\hline 0.90 & $\begin{array}{l}0.8961 \\
0.0013 \\
0.0422\end{array}$ & $\begin{array}{l}0.8962 \\
0.0013 \\
0.0421\end{array}$ & $\begin{array}{l}0.8943 \\
0.0013 \\
0.0412\end{array}$ & $\begin{array}{l}0.8943 \\
0.0013 \\
0.0412\end{array}$ \\
\hline 0.99 & $\begin{array}{l}0.9901 \\
0.0001 \\
0.0042\end{array}$ & $\begin{array}{l}0.9891 \\
0.0010 \\
0.0321 \\
\end{array}$ & $\begin{array}{l}0.9891 \\
0.0002 \\
0.0051 \\
\end{array}$ & $\begin{array}{l}0.9863 \\
0.0017 \\
0.0544\end{array}$ \\
\hline
\end{tabular}




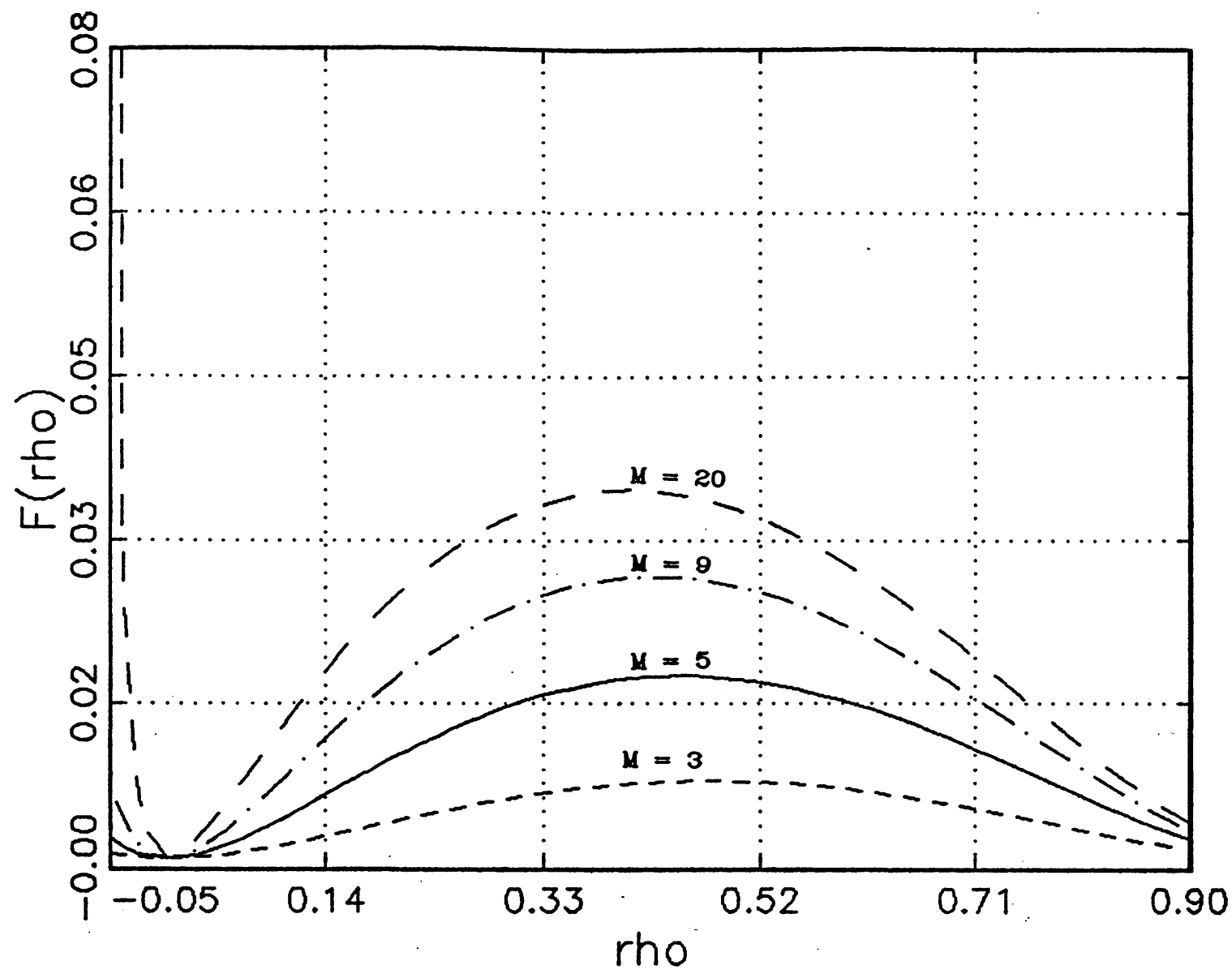

Figure I: Graphs of equation 3.2.7 for $\mathrm{M}=3$, line with short dashes, $\mathrm{M}=5$, the solid line, $M=9$, the line with dots and dashes, and $M=20$, the line with long dashes.

4. DISTRIBUTION OF THE COVARIANCE-VARIANCE RATIO ESTIMATOR, $\hat{\rho}$

\subsection{Introduction}

In this section we consider a model with no block effects and with uniform covariance structure, i.e., the model of Section 3.2, but with equal variances. Similar to (3.2.1) the log-likelihood is given by

$$
\begin{aligned}
\mathrm{L}= & -\frac{\mathrm{N}(\mathrm{M}-1)}{2} \log (1-\rho)-\frac{\mathrm{A}(\mu, \alpha)}{2 \sigma^{2}(1-\rho)} \\
& -\frac{\mathrm{NM}}{2} \log \sigma^{2}-\frac{\mathrm{N}}{2} \log (1+(\mathrm{M}-1) \rho) \\
& +\frac{\mathrm{B}(\mu, \alpha) \rho}{2 \sigma^{2}(1-\rho)(1+(\mathrm{M}-1) \rho)},
\end{aligned}
$$


where $\mathrm{A}(\mu, \alpha)=\sum_{\mathrm{i}=1 \mathrm{j}=1}^{\mathrm{N}} \sum_{\mathrm{ij}}^{\mathrm{M}}\left(\mathrm{Y}_{i j}-\mu-\sigma_{\mathrm{j}}\right)^{2}$ and $\mathrm{B}(\mu, \alpha)=\sum_{\mathrm{i}=1}^{\mathrm{N}}\left(\sum_{\mathrm{j}=1}^{\mathrm{M}}\left(\mathrm{Y}_{\mathrm{ij}}-\mu-\alpha_{\mathrm{j}}\right)\right)^{2}$.

Differentiation with respect to $\sigma^{2}$ and $\rho$ gives the equations

$$
\begin{gathered}
\frac{\partial \mathrm{L}}{\partial \rho}=\frac{\mathrm{NM}(\mathrm{M}-1) \rho}{2(1-\rho)(1+(\mathrm{M}-1) \rho)}-\frac{\mathrm{A}(\mu, \alpha)}{2 \sigma^{2}(1-\rho)^{2}}+\frac{\mathrm{B}(\mu, \alpha)\left(1+(\mathrm{M}-1) \rho^{2}\right)}{2 \sigma^{2}(1-\rho)^{2}(1+(\mathrm{M}-1) \rho)^{2}} \\
\frac{\partial \mathrm{L}}{\partial \rho}=-\frac{\mathrm{NM}}{2 \sigma^{2}}+\frac{\mathrm{A}(\mu, \alpha)}{2 \sigma^{4}(1-\rho)}+\frac{\mathrm{B}(\mu, \alpha) \rho}{2 \sigma^{4}(1-\rho)(1+(\mathrm{M}-1) \rho)}
\end{gathered}
$$

Equating (4.1.2) to zero and solving for $\sigma^{2}$ gives

$$
\hat{\sigma}^{2}=\frac{1}{\mathrm{NM}} \frac{\mathrm{A}(\hat{\mu}, \hat{\sigma})(1+(\mathrm{M}-1) \hat{\rho})-\beta(\hat{\mu}, \hat{\alpha}) \hat{\rho}}{(1-\hat{\rho})(1+(\mathrm{M}-1) \hat{\rho})}
$$

Using $\hat{\sigma}^{2}$ in setting (4.1.1) to zero gives

$$
\hat{\rho}=\frac{1}{M-1} \frac{\sum_{j \neq k}^{M} \sum_{i=1}^{M} \sum_{i j}^{N}\left[Y_{i j}-\bar{Y}_{\cdot j}\right]\left[Y_{i k}-\bar{Y}_{\cdot k}\right]}{\sum_{j=1}^{M} \sum_{i=1}^{N}\left[Y_{i j}-\bar{Y}_{\cdot j}\right]^{2}}
$$

Since $\hat{\rho}$ is the ratio of the average covariance to the average variance, it will be referred to as the covariance-variance ratio estimator. The covariance-variance ratio estimator has appeared in the literature in connection with the estimation of the intraclass correlation (inter-correlation) coefficient, reliability coefficients, and measures of association.

Delury (1938) used the covariance-variance ratio estimator with $M=2$ as an improved estimate of the product moment correlation over the usual sample correlation, if it is assumed that the two varieties have equal variances. Alexander (1947) gave the covariance-variance ratio estimator as one of the estimates of reliability adjusted for trend (the means of the trials are not all equal). Using the method of moments, Coombs (1948) derived the covariancevariance ratio estimator as an estimate of the correlation coefficient. Olkin and Pratt (1958) showed that the unique minimum variance unbiased estimator (UMVUE) of the intraclass correlation is a function of the covariance-variance ratio estimator. Wilks (1961) developed test criteria for testing equality of means, equality of variances and equality of covariances in a normal multivariate population. Wilks's test criteria are functions of the covariance-variance ratio estimator. 
McCulloch (1985) showed that Schluter's variance test, W (given below), for density data is a function of the F-statistic from a two-way analysis of variance (species by samples), with ( $\mathrm{N}-1$ ) and (N-1)(M-1) degrees of freedom, used for testing samples with the species $\mathrm{x}$ samples mean square as an error term. $\mathrm{W}=\mathrm{NV}$ where $\mathrm{V}=\mathrm{M} F /(\mathcal{F}+\mathrm{M}-1)$ and $\mathscr{F}$ is given by

$$
\mathscr{F}=\frac{M(M-1) \sum_{i=1}^{N}\left[\bar{Y}_{i \cdot}-\bar{Y} . .\right]^{2}}{\sum_{j=1}^{M} \sum_{i=1}^{N}\left[Y_{i j}-\bar{Y}_{i \cdot}-\bar{Y}_{\cdot j}+\bar{Y} . .\right]^{2}} .
$$

McCulloch (1985) proposed the covariance-variance ratio estimator as an estimator of the average correlation, and showed that $V=(M-1) \hat{\rho}+1$. This establishes directly the connection between the overall association measure, $\mathrm{V}$, and techniques that examine pairwise species association.

\subsection{Derivation of the density $\hat{\rho}$}

We now derive the distribution of $\hat{\rho}$ when the columns are equally correlated, the variances of the $\mathrm{Y}_{\mathrm{ij}}$ 's are equal and there are no block effects. We also give expressions for the mean and variances in terms of ordinary hypergeometric series.

Equation (4.1.3) can be written as $\hat{\rho}=(\mathscr{F}-1) /(\mathcal{F}+\mathrm{M}-1)$, where $\mathscr{F}$ is given in (4.1.4). For equal correlation and equal variance the distribution of $\mathscr{F}$ is

$$
\mathscr{F} \sim \frac{[1+(\mathrm{M}-1) \rho]}{[1-\rho]} \mathrm{F}_{\mathrm{N}-1,(\mathrm{~N}-1)(\mathrm{M}-1)}
$$

where $F_{k, q}$ represents a variable that has the $F$-distribution with $k$ and $q$ degrees of freedom. With this the density of $\hat{\rho}$ is derived in a straight-forward manner through the cumulative distribution of the F-distribution and is given by 


$$
f_{\hat{\rho}}[x]= \begin{cases}\frac{C\left[\frac{1}{M-1}+x\right]^{t-1}[1-x]^{(M-1) t-1}}{\left[\frac{1}{M-1}+\alpha(1-x)\right]^{M t}} & \frac{-1}{M-1}<x<1 \\ 0 & \text { otherwise }\end{cases}
$$

where $\alpha=\rho /(1-\rho), \mathrm{t}=(\mathrm{N}-1) / 2$ and

$$
C=\frac{\Gamma[M t][1+\alpha M]^{(M-1) t}}{(m-1) M^{M t-1} \Gamma[t] \Gamma[(M-1) t]} \text {. }
$$

For $M=2$ the density of $\hat{\rho}$ reduces to the density Delury (1938) derived.

If $\rho=0,(4.2 .2)$ reduces to the Beta distribution after making the transformation $\mathrm{Y}=((\mathrm{M}-1) \hat{\rho}+1) / \mathrm{M}$. $\mathrm{Y}$ then has the standard form of the Beta distribution with parameters $t$ and (M-1)t. From this the mean of $\hat{\rho}$ is zero and variance is given by

$$
\operatorname{Var}[\hat{\rho}]=[(\mathrm{M}-1)(\mathrm{Mt}+1)]^{-1}=2[(\mathrm{M}-1)(\mathrm{M}(\mathrm{N}-1)+2)]^{-1} .
$$

For the general case, when $\rho$ is not necessarily zero, the mean and variance are functions of $\rho$. Using a change of variable $Y=1-(M-1)(1-X) / M$, the expected value of $\hat{\rho}$ is

$$
\mathrm{E}[\hat{\rho}]=1-\mathrm{C}\left[\frac{(1-\rho) \mathrm{M}}{1+(\mathrm{M}-1) \rho}\right]^{\mathrm{Mt}} \int_{0}^{1} \frac{\mathrm{y}^{\mathrm{t}-1}[1-\mathrm{y}]^{(\mathrm{M}-1) \mathrm{t}}}{[1-\mathrm{Zy}]^{\mathrm{Mt}}} \mathrm{dy}
$$

where $\mathrm{Z}=\mathrm{M} \rho /[1+(\mathrm{M}-1) \rho]$. Denote the ordinary hypergeometric series, (Abramowitz and Stegun, 1972, 15.3.1) as follows

$$
\mathrm{F}[\mathrm{a}, \mathrm{b} ; \mathrm{c} ; \mathrm{Z}]=\frac{\Gamma(\mathrm{c})}{\Gamma(\mathrm{c}) \Gamma(\mathrm{c}-\mathrm{b})} \int_{0}^{1} \frac{\mathrm{y}^{\mathrm{b}-1}[1-\mathrm{y}]^{\mathrm{c}-\mathrm{b}-1}}{[1-\mathrm{Zy}]^{\mathrm{a}}} \mathrm{dy} \text {. }
$$

The series converges when $|\mathrm{Z}|<1$ and $\mathrm{c}>\mathrm{b}>0$; all these are satisfied for the integral in (4.2.3). Substituting the expression for $\mathrm{C}$ and using a linear transformation, the expected value of $\hat{\rho}$ is given by

$$
\mathrm{E}[\hat{\rho}]=1-\frac{[1-\rho]}{[1+(\mathrm{M}-1) \rho]} \mathrm{F}[1,(\mathrm{M}-1) \mathrm{t}+1 ; \mathrm{Mt}+1 ; \mathrm{Z}] \text {. }
$$

The second moment is found similarly and is given by 


$$
\mathrm{E}\left[\hat{\rho}^{2}\right]=1-\frac{2(1-\rho)}{1+(\mathrm{M}-1) \rho} \mathrm{F}^{1}+\left(\frac{1-\rho}{1+(\mathrm{M}-1) \rho}\right)^{2}\left(\frac{1+\frac{1}{(\mathrm{M}-1) \mathrm{t}}}{1+\frac{1}{\mathrm{Mt}}}\right) \mathrm{F}^{2},
$$

where $F^{1}=F(1,(M-1) t+1, M t+1 ; Z)$ and $F^{2}=F(2,(M-1) t+1, M t+2 ; Z) . \quad F^{1}$ and $F^{2}$ and hence $\operatorname{Var}(\hat{\rho})$ can be straightforwardly calculated using the equations (Abramowitz and Stegun, 1972; 15.1.1)

$$
\begin{aligned}
\mathrm{F}(1,(\mathrm{M}-1) \mathrm{t}, \mathrm{Mt}+1 ; \mathrm{Z}) & =\sum_{\ell=0}^{\infty}\left(\prod_{\mathrm{k}=1}^{\ell}\left(1-\frac{\mathrm{t}}{\mathrm{M} t+\mathrm{k}}\right)\right) \mathrm{Z}^{\ell} \\
\mathrm{F}(2,(\mathrm{M}-1) \mathrm{t}+1, \mathrm{Mt}+2 ; \mathrm{Z}) & =\sum_{\ell=0}^{\infty}\left(\prod_{\mathrm{k}=1}^{\ell}\left(1-\frac{\mathrm{t}}{\mathrm{M} t+1+\mathrm{k}}\right)\right) \mathrm{Z}^{\ell}(\ell+1) .
\end{aligned}
$$

For small $M$ and $\rho$ (say $M \leq 10$ and $\rho \leq .5$ ) the above series expansions give three digit accuracy with 100 terms.

We used these expansions to calculate $\operatorname{Var}(\hat{\rho})$ and compare it to the asymptotic variance of $\hat{\rho}$ which is given by

$$
\operatorname{Var}_{\infty}(\hat{\rho})=\frac{2(1-\rho)^{2}(1+(\mathrm{M}-1) \rho)^{2}}{\mathrm{NM}(\mathrm{M}-1)}
$$

Table II shows the calculations for several values of $M, \rho$ and $N$. The asymptotic approximation is quite good for small $\mathrm{M}$ and $\rho$, but requires very large sample sizes for larger $M$ and/or $\rho$. 


\section{TABLE II}

$\operatorname{Var}(\hat{\rho}), \operatorname{Var}_{\infty}(\hat{\rho})$ and $\operatorname{Var}(\hat{\rho}) / \operatorname{Var}_{\infty}(\hat{\rho})$ for $\operatorname{Various} M, \rho$ and $\mathrm{N}$

\begin{tabular}{|c|c|c|c|c|}
\hline \multirow[b]{2}{*}{$\mathrm{N}$} & \multicolumn{2}{|c|}{$M=5$} & \multicolumn{2}{|c|}{$\mathrm{M}=10$} \\
\hline & $\rho=.3$ & $\rho=.7$ & $\rho=.3$ & $\rho=.7$ \\
\hline \multirow[t]{2}{*}{5} & $.0474^{1} .0495^{2}$ & $.0260 \quad .0458$ & .0298 .0305 & $\begin{array}{ll}.0213 & .0362\end{array}$ \\
\hline & $.96^{3}$ & .57 & .98 & .59 \\
\hline \multirow[t]{2}{*}{15} & $.0158 \quad .0161$ & .0087 .0108 & $\begin{array}{ll}.0099 & .0100\end{array}$ & $.0071 \quad .0088$ \\
\hline & .98 & .80 & .99 & .81 \\
\hline \multirow[t]{2}{*}{35} & $.0068 \quad .0068$ & $.0037 \quad .0041$ & $.0043 \quad .0043$ & $.0030 \quad .0033$ \\
\hline & .99 & .91 & .99 & .91 \\
\hline \multirow[t]{2}{*}{75} & $.0032 \quad .0031$ & $.0017 \quad .0018$ & $.0020 \quad .0020$ & $.0014 \quad .0015$ \\
\hline & .99 & .95 & 1.00 & .96 \\
\hline \multirow[t]{2}{*}{155} & $.0015 \quad .0015$ & $.0008 \quad .0008$ & $.0010 \quad .0010$ & $.0007 \quad .0007$ \\
\hline & 1.00 & .98 & 1.00 & .98 \\
\hline
\end{tabular}




\section{BIBLIOGRAPHY}

Abramowitz , M. and Stegun, I.A. (1972). Handbook of Mathematical Function Formulae, Graphs and Mathematical Tables. National Bureau of Standards, Applied Mathematics Series, No.55.

Alexander, H. W. (1947). "The estimation of reliability when several trials are available," Psychometrika, 12: 79-99.

Andersen, A. H, Jensen, E. B., and Schou, G. (1981). "Two-way analysis of variance with correlated errors," International Statistical Review, 49: 153-167.

Box, G.E.P. (1954). "Some theorems on quadratic forms applied in the study of analysis of variance problems, I. Effects of inequality of variances in the one-way classification, II. Effects of inequality of variance and correlation between errors in the two- way classification," Annals of Mathematical Statistics, 25: 290-302, 484-498.

Coombs, C. H. (1948). "The role of correlation in analysis of variance," Psychometrika, 13: 233-243.

Craig, A. T. (1943). "Note on the independence of certain quadratic forms," Annals of Mathematical Statistics, 14: 195-197.

Delury, D. B. (1938). "Note on correlations," Annals of Mathematical Statistics, 9: 149-151.

Donner, A. (1986). "A Review of inference procedures for the intraclass correlation coefficient in a one-way random effects model". International Statistical Review, 54: 67-82.

Edlefsen, L. E., and Jones, S. D. (1987). Gauss version 1.49b, Aptch Systems, Inc. Kent, WA.

Graybill, A. F. (1983). Matrices with Applications in Statistics. Second Edition. Wadsworth. McCulloch, C.E. (1985). "Variance tests for species association," Ecology, 66: 1676-1681.

Olkin, I., and Pratt, W. J. (1958). "Unbiased estimation of certain correlation coefficients," Annals of Mathematical Statistics, 29: 201-211. 
Olkin, I., and Vaeth, M. (1981). "Maximum likelihood estimation in two-way analysis of variance with correlated errors in one way classification," Biometrika, 68: 653-660.

Schluter, D. (1984). "A variance test for detecting species association, with some example applications," Ecology, 65: 998-1005.

Searle, S.R. (1971). Linear Models. New York: John Wiley and Sons.

Searle, S.R. (1984). Matrix Algebra Useful for Statistics. New York: John Wiley and Sons.

Wilks, S.S. (1946). "Sample criteria for testing equality of means, equality of variances and equality of covariances in normal multivariate distributions," The Annals of Mathematical Statistics, 17: 257-281. 